\title{
Homeopathic treatment of infertility: a medical and bioethical perspective
}

\begin{abstract}
Homoeopathy has a significant clinical history, tracing its roots back to the ancient physician Hippocrates. In the last 30 years, it has surged in popularity among those seeking alternative forms of medical treatment. Countering this increase in popularity, some recent meta-analyses have suggested that the therapeutic claims of homeopathy lack scientific justification. A popular area of homeopathic application is infertility. In this paper we review the homeopathic approach and the bioethical implications to infertility, and propose that monitoring the effects of homeopathic remedies on infertile women may be as effective method to assess the efficacy of this form of alternative medicine.
\end{abstract}

Keywords: homeopathy, alternative medicine, infertility, ethical issues
Volume 5 Issue 5 - 2017

\author{
Domenick J Masiello, John D Loike \\ Department of Physiology and Cellular Biophysics, Columbia \\ University College of Physicians and Surgeons, USA
}

Correspondence: John D. Loike, Department of Physiology and Cellular Biophysics, and Center for Bioethics, Columbia University College of Physicians and Surgeons, 630 West 168th Street, New York, NY 10032, USA, Email jb15@columbia.edu

Received: November 13, 2016 | Published: March 06, 2017

\section{Introduction}

Homeopathic medicine is an alternative medical system was first practiced in Germany more than 200years ago. Developed by its founder Dr. Samuel Hahnemann, homeopathic treatment is based on two principles. The first specifies that "like cures like"- a disease can be cured by a substance that produces similar symptoms in healthy people. The second principle, commonly referred to the "law of minimum dose" stipulates that very low doses of toxic materials can treat specific symptoms. Homeopathic remedies are composed of a variety of substances derived from plants, minerals, animals, or even potentially toxic materials. The FDA has regulated the use of homeopathic drugs in the United States since 1938, but does not evaluate the remedies for safety or effectiveness. ${ }^{1}$

In this article, we examine the application of homeopathy to medical infertility, a topic that has received little attention in medical journals. ${ }^{2}$ We first review how homeopathy addresses and provides potential treatments for various medical conditions related to infertility. We then discuss some of the bioethical concerns related to homeopathy, specifically its efficacy in treating infertility issues. One controversial issue in homeopathy is the lack of acceptable scientific validity in assessing efficacy of homeopathic remedies. We propose that assessing and monitoring the percent of infertile patients that get pregnant within 2 years of homeopathic treatments may provide a scientifically valid method to examine the success rate of homeopathic interventions in achieving pregnancies.

\section{Homeopathic analysis of disease and symptoms}

The overall outcome of homeopathy is to determine a specific course of treatment for each patient by following an established protocol for diagnosis. First, the homeopathic physician identifies the cause of the illness, such as infertility, by compiling a comprehensive medical history of the patient, focusing on patient symptoms and inquiring about factors that may aggravate or ameliorate the symptoms. This analysis includes identifying daily times when symptoms are presented and what conditions exacerbate or ameliorate these symptoms. Some symptoms as described by the patient can be uncommon, such as "a headache presented as a sensation that the brain is rocking back and forth inside the skull"; abdominal pain in which "something alive is crawling around inside". These uncommon symptoms, known in homeopathy as strange, rare and peculiar, oftentimes prove to be the most useful in relaying medical information to the homeopathic physician.

Other critical pieces of information that the homeopath needs to obtain include life-style characteristics of the patient such as food preferences, weather preferences and several other inclinations. Finally, the physician performs a physical exam and obtains past medical history including diagnostic tests, past medications and other non -drug therapies that the patient is taking. He also obtains a familial, social, and occupational history of the patient.

The symptoms of the patient are then matched as closely as possible to those listed in a 200year- old homeopathic computerized compendium of symptoms arranged by anatomical location referred to as a repertory. These computer databases allow the homeopathic physician to effectively process data by combining rubrics and weighing symptoms in order to account for patient emphasis and eliminating classes of remedies. These computer programs will also generate a chart of likely remedies, and prioritize them in a hierarchal manner, based on the strategic criteria selected. The physical appearance, personality, and behavior of the patient, are also important components of the analysis that cannot be directly analyzed by the computer. During the next step of the analysis, the homeopath assesses whether the symptoms cluster as primarily mental, emotional or physical components of an ailment. This interpretive material is holistically synthesized when choosing an appropriate remedy and formed by combining physiognomy, personality, reported symptoms, traits and generalities. Once a remedy is selected, the homeopath may assess the potency of the remedy that the patient must take.

\section{Specific infertility treatments using homeopathy}

The homeopath first makes the analysis whether infertility is caused by symptoms of the man, woman or both. In this paper we focus on the symptoms of the female partner. Based on the symptoms described by the woman, the homeopath first attempts to improve the patient's overall health to allow for self-healing, especially in women who experience mental and emotional distress. In one actual case, a twenty-nine year old female patient sought homeopathic infertility treatment after two years of failure to become pregnant. The patient was a tall, large, phlegmatic woman with a fair complexion and a 
dislike for cold and damp weather. She complained about constipation and low stamina, and fatigue. Her personality reflected both a sense of stubbornness and a caring sensitivity to others' criticisms and suffering, with a childlike innocence about her. As a child she suffered from febrile seizures that were sometimes associated with middle ear infections. As an adult, her menstrual periods lasted 6-7days and were described as heavy and associated with lower back and thigh pain She has never contracted any sexually transmitted disease and only used barrier methods for contraception. Based on her personality and physical symptoms, she was prescribed Calcarea Carbonica 200C to stimulate her overall health and self-healing. Four weeks later, she reported that she had increased energy, improved bowel movements, and less painful menstrual pains. The potency of her dose of Calcarea Carbonica was reduced to $1000 \mathrm{c}$ and she was told to return two months later. At the second follow-up visit, her stamina and energy continued to improve and she lost ten pounds. The dose of Calcarea Carbonica was further decreased to $10,000 \mathrm{C}$ and told to return in 3 months. Six weeks later, she reported that she was pregnant.

Most cases of infertility are significantly more complex than the one previously described, rendering it difficult for a homeopath to immediately decide upon a remedy. Often, remedies are selected to address specific reproductive symptoms and issues, rather than solely promote self-healing. In the Female Genitalia/Sex Chapter of the repertory in Homeopathy, "Sterility" is a large rubric consisting of 110 remedies. These remedies can be constituted from a diverse array of products such as plants, animals, and minerals. In addition, this rubric may contain substances derived from animal organs (usually sheep) such as pituitary glands, anterior pituitary glands and ovaries. Furthermore, homeopaths also prescribe remedies made from pathological tissue or pathogenic microorganisms such as syphilis, gonorrhea, and Chlamydia. It is useful for the homeopathic physician to consider the etiology of infertility from several different sources, including tubal, ovarian, endometrial, uterine, cervical, or age-related causes.

During the next step of the analysis, the homeopath takes into account whether the patient has undergone an extensive gynecological analysis in other clinics, as he believes that such treatments may affect the course of the homeopathic treatment. For example, the patient may have had hormonal treatments based on their levels of FSH, $\mathrm{LH}$, progesterone, estradiol, and prolactin. Many female patients have undergone a hysterosalpingogram, to search for fallopian tube scarring or abnormalities. Unlike the case mentioned above, the symptoms of complex cases may actually represent multiple disease states within the same individual, or may represent previous illnesses whose treatment was suppressive and whose sequelae may still be present, albeit in subtle form. Hence, there may be multiple layers, each with a complex array of symptoms.

If the patient's complaints, symptoms and characteristics are mostly physical in nature, then the results of laboratory and radiological assessment gain increased relevance. For instance, if there is a history of sexually transmitted disease, the homeopath may opt to treat the patient with a remedy made from the causative bacterium, such as Chlamydia trachomatis . Even though a patient with gonorrhea was previously treated with antibiotics, prescribing a dose of Medorrhinum $10 \mathrm{M}$ (gonococcus) can improve the fertility potential of the patient. In patients exhibiting a history of pelvic inflammatory disease with tubal inflammation the homeopathic physician might prescribe remedies such as Aconite, Apis, Bacillinum, and other remedies for scarring (cicatrix). If endometriosis is an issue, then rubrics for menstrual irregularities can be added. In the case of polycystic ovary disease, the remedy rubric includes Apis, Bovista, Bufo, Cantharis and Oophorinum.

Occasionally, homeopaths may choose to treat infertility by attempting to heal a specific organ. For instance, in the case where birth control contraceptive medication resulted in a pituitary adenoma with concurrent elevated prolactin levels, the homeopath might treat the side effects of contraceptive medication by administering a remedy made from the birth control pill or one of its constituents. This is referred to as a non-traditional isopathic approach to clear the longterm effects of conventional hormonal manipulation.

In some patients, long-term birth control can result in primary ovarian failure and the homeopath chooses a remedy to improve ovarian function. The homeopath believes that Agnus Castus (Chaste Tree) will help normalize reproductive function via the pituitary by balancing FSH and LH levels. Sabal Serrulata will help in cases of polycystic ovary syndrome by reducing excessive androgens. The homeopath may choose a combinatorial approach by prescribing remedies that address overall health of the patient and also remedies directed to specific organs. Homeopaths believe that their ability to treat various syndromes lies in homeopathy's foundation, a belief in the vital force, an energy that is present in all living beings and maintains internal homeostasis.

Combining the homeopathic approach together with a conventional western-medical approach is also a method used by homeopaths to treat infertility. It is acceptable to treat infertility with homeopathic remedies during the early diagnostic phase of the conventional approach. However, more involved fertility treatments such as in vitro fertilization may be incompatible with homeopathic prescribing.

\section{Ethical and regulatory issues regarding homeopathy}

There are two related bioethical issues surrounding homeopathy. The first is the lack of scientific evidence in understanding the mechanism by which homeopathic remedies improve the health of the patient. ${ }^{3}$ Homeopathy is often viewed as a controversial, unscientific medical modality, often sought by those who have not been able to find effective treatment for chronic symptoms using conventional medicine. In most cases, the patient receives a remedy that has been diluted to such an extent that it is chemically composition is water without any active pharmacological agent. The second issue is the homeopathic principle that the vital force of a person impacts homeopathic remedies. How does "vital force" translate into physiological mechanisms? Modern healthcare professionals, in accordance with the Hippocratic Oath and established bioethical principles, have an obligation to provide treatment that is scientifically valid and based on experiment evidence of efficacy. The lack of hard-core scientific evidence and the reliance on undefined vital forces raises major ethical concerns in utilizing homeopathy. Moreover, it is important to consider that among US adults, $2.1 \%$ used homeopathy within the past 12 months and treating respiratory and otorhinolaryngology complaints were most commonly treated $(18.5 \%) .{ }^{4}$ These issues underscore a critical question is how should society integrate "common knowledge" and "anecdotal experiences" that goes against existing scientific dogma?

Though the mechanisms by which homeopathic treatments affect human health remains unknown, numerous articles have suggested that homeopathy responses operate via a placebo effect. The phrase Primum non nocere, a Latin expression meaning "First do no harm," is a fundamental principle of Western medical care. There is little evidence that homeopathy causes any serious side effects. Some physicians believe homeopathy treatment to have a placebo effect and 
believe that it should be continued. Additionally, they assert that if the results are similarly effective in both traditional treatments, and homeopathic treatments, than homeopathic treatment could be utilized as a viable alternative. In fact, an estimated $30 \%$ of men presenting for infertility evaluation use alternative therapies. ${ }^{5}$ However, the ethics of placebo research underline that placebos should not be used when a patient's life is at risk. Physicians do not generally utilize placebo pills for serious diseases that can compromise the life of a patient. In this case, while homeopathic treatment might be utilized for infertility, it would not be suitable for many disorders.

There is a need to identify the underlying mechanisms of homeopathic remedies. Many scientists believe that homeopathy operates through a placebo effect. ${ }^{6}$ However, several studies present data that homeopathic remedies are not operating though a placebo response. Several reports, for example, show that homeopathic remedies are effective in treating infants, comatose patients and animals $^{7-13}$ induce oestrus in cows. ${ }^{14}$ Another report has shown that homeopathic remedies might be trigger cytolytic activity in breast cancer cells maintained in culture.$^{15}$ Finally, as placebos are generally effective within a few weeks and homeopathic remedies may take several months to work, support the concept that the underlying mechanism of homeopathy is not a placebo effect. In addition, there are few studies that examine potential side effects of homeopathy. One study describes homeopathic aggravations as an important consideration in assessing medical side effects of this treatment modality.

There are several reasons for the lack of comprehensive studies on the effects of homeopathy. Current medical procedures and the pharmaceutical industry are rigorously based on evidence and experimentation in order to develop a treatment that is widely applicable to the general population Homeopathy is a highly individualized method tailored to the personal needs of the patient. Balancing these two semi-opposing schools of thought has proven very difficult for those clinicians who want to examine the efficacy of homeopathy in well-designed studies for infertility. This is partly because in homeopathy, each patient may receive a different remedy for a classically defined disease. Thus, obtaining a population of volunteers who are being treated for one particular "disease" is difficult. Additionally, the symptom- and patient personality- based analysis in selecting the appropriate remedy renders it difficult to recruit a large number of volunteers expressing the same symptoms or undergoing the same remedies, while still controlling for a doubleblind clinical trials.

In light of these concerns, scientists and bioethicist should consider assessing the efficacy of homeopathy by systematically interviewing patients who have undergone homeopathy and their views on the efficacy of such treatments. Such studies cannot be double-blinded or controlled as in classical clinical trials. Moreover, interpreting their results is scientifically challenging. We propose that homeopathic treatments for infertility offer an added advantage with regards to patient clinical outcomes by asking whether the patient undergoing infertility treatments became pregnant within a year or two after homeopathy treatment began. Recognizing that such a study is based on the testaments of patients but still may provide scientific validity on the medical benefits of homeopathy. Most studies underline that there is insufficient evidence to make a conclusive decision regarding its efficacy. ${ }^{16}$ The fact that millions believe in homeopathy is also not in itself scientific proof of its efficacy. Rather, it would be a great service if we gained a better understanding of this alternative medical treatment by trying to design the best and most holistic study possible.

\section{Government regulations}

Government allocation for homeopathic treatment for infertility is another ethical/regulatory issue that needs clarification. In the United States, little government money is spent on homeopathic research. As discussed above, $2.1 \%$ of Americans use homeopathy and pay billions of dollars for homeopathic medicines and \$170million for visits to homeopathic practitioners ${ }^{17}$. Opponents of homeopathy protest the funneling of public funds for homeopathy, as it has not been wholeheartedly accepted in the scientific community. However, the situation differs in Europe as several countries have contributed the national budget towards homeopathic treatments. For example, the Swiss government has recently urged the inclusion of homeopathy and complementary and alternative medicine in their national health program. ${ }^{18}$ It is unclear if these European governments will continue to support homeopathy as a report of the Science and Technology Committee of the United Kingdom House of Commons ${ }^{19}$ made known its intent to halt NHS funding of homeopathy.

\section{Conclusion}

In general, homeopathy is viewed as unproven science, yet it may be considered "good medicine". ${ }^{20}$ While there is a clear need to conduct more clinical trials on the efficacy of homeopathy, there are a variety of barriers that have limited initiating such trials. Using homeopathy to treat infertility may offer a unique opportunity for assessing its effectiveness with an established outcome (i.e., pregnancy). With respect to treating infertile couples, the homeopathic physician also should try to provide a time line when the couple can expect to get pregnancy and what are the alternatives if pregnancy is not achieved. In the long-term, homeopaths can follow specific measures in order to raise the bioethical standards of their treatments to match that of traditional westernized treatment. Generally, the homeopathy physician does not publish his or her results, nor does he replicate the same technique of treatment for patients with the same disorder. O'Donovan et al., ${ }^{21}$ conducted a meta-analysis of homeopathic trials for infertility, and concluded that the quality of the majority of trials was poor. Furthermore, it is difficult to evaluate the results of homeopathic treatments in the same manner as conventional treatment as a result of the differing medical principles and differing healing processes underlying each method..$^{22}$ However, if the homeopath publishes and evaluates the results of infertility treatments, he or she can procure a definitive result of the efficacy of their methods. Rather than have anecdotal evidence, homeopaths can have a success rate measured in fertility, and compare it with other more mainstream treatments for infertility. By comparing two disparate methods and measuring the success right, researchers might be able to definitively conclude the value of homeopathy. ${ }^{23}$

As with infertile couples, homeopathic physicians should apply bioethical principles by providing any patient with an honest informed consent document. A homeopathic physician should ensure that the patient is aware of the risks of utilizing homeopathic medicine as well as alternative medical procedures that are available. The patient must be cognizant that there is no clear scientific understanding of how homeopathic remedies work and it is only through the testaments of others that supports any clinical efficacy of homeopathy. It is only by having an educated patient and a conscientious homeopath that that it is possible to ensure the full autonomous right of a patient to choose whether homeopathic approach for their infertility is appropriate.

\section{Acknowledgments}

None. 


\section{Conflicts of interest}

Author declares there are no conflicts of interest.

\section{Funding}

None.

\section{References}

1. ht t p ://ww w fda.gov/i c e c i/compliancemanuals / compliancepolicyguidancemanual/default.htm

2. Kalampokas T, Botis S, Kedikgianni-Antoniou A, et al. Homeopathy for infertility treatment: a case series. Clin Exp Obstet Gynecol. 2014;41(2):158-159.

3. Fisher P, Ernst E. Should doctors recommend homeopathy? BMJ. 2015;351:h3735.

4. Dossett ML, Davis RB, Kaptchuk TJ, et al. Homeopathy Use by US Adults: Results of a National Survey. Am J Public Health. 2016;106(4):743-745.

5. Zini A, Fischer MA, Nam RK, et al. Use of alternative and hormonal therapies in male infertility. Urology. 2004;63:141-143.

6. Segar J . Complementary and alternative medicine: Exploring the gap between evidence and usage. Health. 2012;16(4):366-381.

7. Almeida L, Campos M, Herrera H, et al. Effects of homeopathy in mice experimentally infected with Trypanosoma cruzi. Homeopathy. 2008;92(2):65-69.

8. Aziz D, Schnurrbusch U, Enbergs H. Effects of two homeopathic complexes on bovine sperm mitochondrial activity. Homeopathy. 2012;101(2):99-102.

9. Bornhöft G, Wolf U, Ammon K, et al. Effectiveness, Safety and CostEffectiveness of Homeopathy in General Practice - Summarized Health Technology Assessment. Forschende Komplementmed. 2006;13supp12:19-29.

10. Lobreiro J. Homeopathic treatment for infertility in a prize Nelore bull. Homeopathy. 2007;96(1):49-51.
11. Mathie RT, Van Wassenhoven M, Jacobs J, et al. Model validity and risk of bias in randomised placebo-controlled trials of individualised homeopathic treatment. Complement Ther Med. 2016;25:120-125.

12. Soto F, Vuaden E, Coelho C, et al. A randomized controlled trial of homeopathic treatment of weaned piglets in a commercial swine herd. Homeopathy. 2008;97(4):202-205.

13. Torbicka E, Brzozowska A, Wylczynski J. RSV infections in infants: therapy with a homeopathic preparation. Biomedical Therapy. 1998;16:256-260.

14. Rajkumar R, Srivastava S, Yadav M, et al. Effect of a Homeopathic complex on oestrus induction and hormonal profile in anoestrus cows. Homeopathy. 2006;95(3):131-135.

15. Preethi K, Ellanghiyll S, Kuttan G. Induction of Apoptosis of Tumor Cells by Some Potentiated Homeopathic Drugs: Implications on Mechanism of Action. Integr Cancer Ther. 2011;11(2):172-182.

16. Linde $\mathrm{K}$, Clausius N, Ramirez $\mathrm{G}$, et al. Are the clinical effects of homeopathy placebo effects? A meta-analysis of placebo-controlled trials. Lancet. 1997;350(9081):834-843.

17. Nahin R, Barnes P, Stussman Bl. Costs of Complementary and Alternative Medicine (CAM) and Frequency of Visits to CAM Practitioners: United States, 2007. National health statistics repor. 2007.

18. http://www.huffingtonpost.com/dana-ullman/homeopathic-medicineb_1258607.html

19. http://www.parliament.uk/business/committees/committees-archive/ science-technology/s-t-homeopathy-inquiry/

20. Spence D. Good medicine: homeopathy. BMJ. 2012;345:e6184.

21. O'Donovan P, Vandekerckhove P, Lilford R. Infertility: Treatment of male infertility: is it effective? Review and meta-analyses of published randomized controlled trials. 1993; 8:209-1222.

22. Oberbaum M, Vithoulkas G, Van Haselen R. Clinical Trials of Classical Homeopathy: Reflections on Appropriate Research Designs. J Altern Complement Med. 2003;9(1):105-111.

23. Carter B. Methodological issues and complementary therapies: researching intangibles? Complement Ther Nurs Midwifery. 2003;9(3):133-139. 